\title{
APPLIANCES
}

\section{ANGLE OF THE ANTERIOR CHAMBER VIEWED BY TRANSMITTED LIGHT*}

BY

\section{J. H. DOBREE and E. F. FINCHAM}

From the Institute of Ophthalmology, London

THE viewing of translucent structures by transmitted light is a well established practice in ophthalmology, but so far does not seem to have been applied to the region of the angle of the anterior chamber. A simple and effective method of transillumination has been devised, and, as certain structures in this important region show to better advantage under these conditions, the technique is presented and some preliminary observations on about 100 eyes recorded.

\section{METHOD}

A minute endoscopy lamp (2.5.-v. cystoscope lamp made by Rimmer) lies in a perspex lamp holder which has a narrow hole bored along its entire length (Fig. 1). The bulb almost touches the rim of a Goldmann gonioscope, and the small air-space surrounding it prevents excessive heating. The base of the lamp is connec-

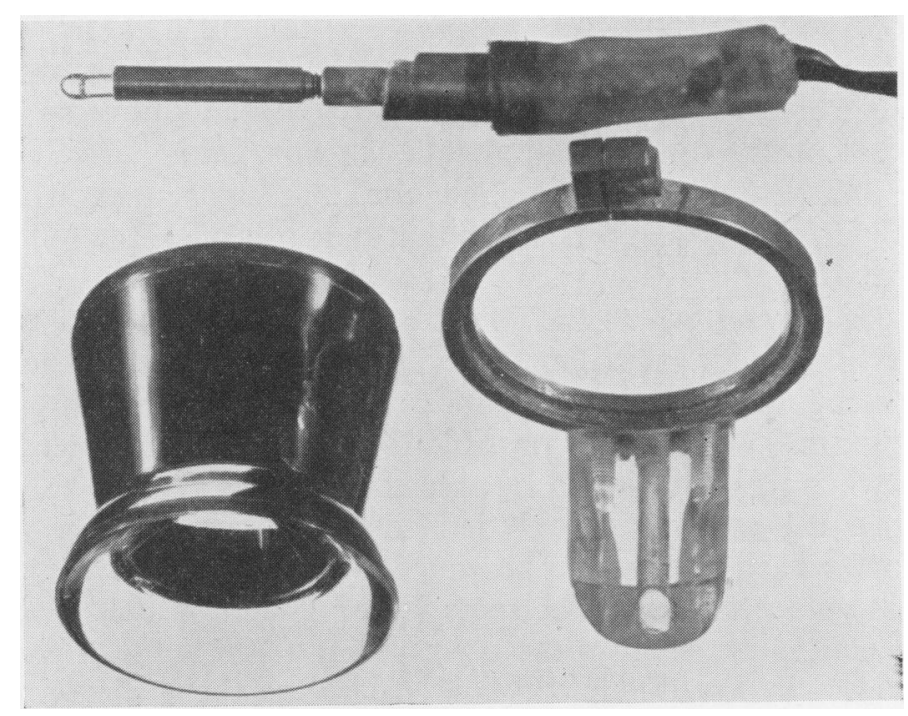

Fig. 1.-Component parts.

*Received for publication August 10, 1953. 
ted to a 3-v. battery by two fine wires. The apparatus is fixed on to the gonioscope by a metal collar with the lamp opposite the viewing mirror (Fig. 2).

In most instances, the gonioscope, with the transilluminating system attached, was used in conjunction with a modern Zeiss slit-lamp so that any particular field could be illuminated, either by transmitted light using the new attachment or by reflected light through the orthodox illuminating system of the slit-lamp, and, by simply switching from one system to the other, a direct comparison of the two methods of illumination could be made.

APPEARANCE OF THE NORMAL ANGLE SEEN BY TRANSMITTED LIGHT

The intensity of illumination obtained by the system described is unexpectedly brilliant, being approximately equal to that obtained with a 3-mm. wide slit-lamp beam. The bulb throws a diffuse patch of light over several square millimetres of the limbus so that an arc of about $60^{\circ}$ of the angle is illuminated in any field. Furthermore, this spread of light was found to include the trabecular area in eyes having abnormally large or small corneae, so that the position of the bulb is effective for any size of eye.

The tissues through which the light passes contain many blood vessels and these impart a somewhat red hue to the picture. A more striking difference however is the fact that the cornea and sclera are rendered more transparent by transillumination, and it is possible to see further into their depth than is the case when they are viewed by reflected light.

The trabecula is particularly well defined and shows up as a honey-coloured band of somewhat gelatinous appearance in sharp contrast with the cornea, which takes on a pearly-white colour. Any pigment on the trabecula stands out in excellent contrast from the surrounding tissues, and pigment patches often stand out in a well-defined plane, clearly on the surface rather than in the substance of the tissue.

The vessels in the vicinity of the angle, which are almost invisible by the usual method of illumination, are shown up to advantage by trans- 
illumination. Blood filling the canal of Schlemm is easy to identify and corneal capillaries can often be seen in the depths of the tissue extending beyond Schwalbes line towards the centre of the cornea. Larger vessels lying near the limbus, such as forwardly placed anterior ciliary arteries, can sometimes be plainly seen, looping deeply to disappear behind the trabecula in the depths of the angle. Observation of these vessels is further facilitated by the incorporation of a yellow/green filter in the viewing system.

\section{Some Appearances seen in Pathological ANgles viewed BY TRANSMITTED LIGHT}

(a) Iris Inclusions.-Iris tissue lying in the substance of the cornea and sclera is usually invisible when viewed by the orthodox method. When seen by transmitted light, however, iris inclusions, whether caused by operation or trauma, are much more readily visible and their size and extent can often be made out. It is thought that extensions into the fibrous tunic from malignant melanomata of the iris or ciliary body will be found to show up in much the same way.

(b) Narrow Angles.-The apex of a narrow angle, which usually appears as a dark cleft when viewed by the orthodox method, is easier to see when illuminated through the cornea. The position of the anterior edge of the trabecula and the presence of synechiae within a narrow angle are often easier to identify by this method.

(c) Peripheral Anterior Synechiae.-Transillumination often shows these structures to be of a very much looser and more spongy texture than they appear when seen by reflected light. Some of the less pigmented types are, however, less clearly seen by transillumination, since, being rendered more translucent, they are not so well defined as when they are seen by reflected light.

\section{COMMENT}

The main disadvantages inherent in illuminating the angle by a bulb attached to the gonioscope are that a certain amount of heat is generated and that the leads are an additional encumbrance. Patients often experience a sensation of warmth and it is our practice to switch off the current for 10 seconds in every minute to allow the heat to disperse, but as no thermal reaction in the conjunctiva has been encountered even this may be an unnecessary precaution.

Although the leads are found to be a disadvantage when they are used in conjunction with a slit-lamp, it must be pointed out that the observer is entirely independent of any rigid system of illumination (or for that matter of a fixed magnifying system) and neither slit-lamp beam not deviating prism is required. Furthermore, the attachment can be used with the patient in any position and it could easily be adapted to devices in use for goniotomy.

We are indebted to Messrs. N. Roberts and F. H. Sheen for constructing the apparatus and to the Department of Medical Illustration, Institute of Ophthalmology, for the photographs. 